\title{
O enfermeiro na assistência ao recém- nascido: conhecimentos, habilidades e atitudes adicionais
}

\author{
Ana Leticia Monteiro Gomes*, Cristiane Rodrigues Rocha, D.Sc.**, Maria Antonieta Rubio Tyrrell, D.Sc.***
}

\begin{abstract}
* Enfermeira, Residente de Enfermagem da Secretaria Municipal de Saúde em convênio com a Universidade Federal do Estado do Rio de Janeiro/Escola de Enfermagem Alfredo Pinto, ${ }^{* *}$ Enfermeira, Especialista em Enfermagem Obstétrica, Universidade Federal do Rio de Janeiro/Escola de Enfermagem Anna Nery, Professora Adjunta da Universidade Federal do Estado do Rio de Janeiro/Escola de Enfermagem Alfredo Pinto, *** Graduação em Revalidação do Titulo de Graduação de Enfermagem na Universidade Federal do Rio de Janeiro/Escola de Enfermagem Anna Nery, Especialista em Enfermagem Obstétrica e em Enfermagem Pediátrica, Universidade Federal do Rio de Janeiro/Escola de Enfermagem Anna Nery, Professora Titular da Universidade Federal do Rio de Janeiro/Escola de Enfermagem Anna Nery
\end{abstract}

\section{Resumo}

O objetivo do estudo foi analisar os conhecimentos e habilidades adicionais dos enfermeiros que atuam no atendimento ao recém-nascido no município do Rio de Janeiro. O método foi quantitativo, transversal, não-experimental, descritivo, com técnica de amostragem por conglomerados. Os dados foram processados com o auxílio do Microsoft Office Excel 2007 e analisados à luz de documentos e manuais da Confederação Internacional das Parteiras e do Ministério da Saúde. Os resultados mostram que 41,66\% dos enfermeiros têm todos os conhecimentos adicionais avaliados e 33,33\% têm todas as habilidades adicionais. Concluímos que o enfermeiro desempenha seu papel de educador com propriedade, no entanto, a ausência de alguns conhecimentos adicionais pode conferir uma habilidade equivocada e uma prática profissional que confere um risco à assistência ao recém-nascido, devendo o profissional ter uma avaliação admissional e um treinamento prévio que assegure sua competência.

Palavras-chave: competência profissional, recém-nascido, assistência de enfermagem, atenção básica.

\section{Abstract}

\section{The nurse in caring for the newborn: additional knowledge, skills and attitudes}

The aim of the study was to analyze the additional knowledge and skills of nurses working in the care of newborns in the city of Rio de Janeiro. The method was quantitative, cross-sectional, non-experimental, descriptive, using a conglomerate sampling technique. The data were processed with the help of Microsoft Office Excel 2007 and were analyzed in the light of documents and manuals of the International Confederation of Midwives and the Ministry of Health. The results show that $41.66 \%$ of nurses have all the evaluated additional knowledge and $33.33 \%$ have all the additional abilities. We 
concluded that the nurses play the role of educators properly. However, the absence of some additional knowledge can lead to misguided abilities and to a professional practice that confers a risk in newborn care, therefore the professionals should undergo an admission assessment evaluation and prior training in order to ensure their competence.

Key-words: professional competence, newborn, nursing care, primary health care.

\section{Resumen}

\section{El enfermero en el cuidado del recién nacido: conocimientos, habilidades y actitudes adicionales}

El objetivo del estudio fue analizar los conocimientos y habilidades adicionales del enfermero que trabaja en el cuidado de los recién nacidos en la ciudad de Río de Janeiro. El método es cuantitativo, transversal y no experimental, descriptivo, con la técnica de muestreo por conglomerados. Los datos fueron procesados con la ayuda de Microsoft Office Excel 2007 y fueron analizados a la luz de los documentos y manuales de la Confederación Internacional de Matronas y el Ministerio de Salud. Los resultados muestran que el $41,66 \%$ de los enfermeros tienen los conocimientos adicionales evaluados y $33,33 \%$ tienen todas las habilidades adicionales. Llegamos a la conclusión de que el enfermero desempeña su papel de educador con propiedad, sin embargo, la ausencia de algunos conocimientos adicionales puede conferir una habilidad equivocada y una práctica que confiere un riesgo en la atención del recién nacido, debiendo el profesional hacer una evaluación de ingreso y una formación previa para asegurar su competencia.

Palabras-clave: competencia profesional, recién nacido, cuidados de enfermería, atención básica.

\section{Introdução}

Esta pesquisa foi desenvolvida em 2011, no Núcleo de Pesquisa em Saúde da Mulher (NUPEM) da Escola de Enfermagem Anna Nery (EEAN) da Universidade Federal do Rio de Janeiro (UFRJ) em parceria com o grupo de pesquisa Cuidado e Competência dos Profissionais de Enfermagem na Saúde da Mulher, criado e cadastrado em 2009 na Universidade Federal do Estado do Rio de Janeiro.

No sentido de garantir o entendimento dos leitores acerca das competências adicionais, é importante explicar que, no ano de 2002, a Confederação Internacional das Parteiras (ICM), em parceria com a Organização Mundial de Saúde (OMS) e a Federação Internacional de Ginecologia e Obstetrícia (FIGO), realizou um estudo envolvendo 17 países dos 05 continentes sobre os conhecimentos, habilidades e atitudes que os profissionais de saúde precisam para realizar a assistência à mulher no ciclo gravídico-puerperal. Este estudo resultou no documento sobre as Competências Essenciais para o Exercício Básico da Obstetrícia [1]. Tal documento registrava lacunas quanto aos dados no Brasil.

Assim, estas lacunas justificam o presente estudo, que contemplou as competências adicionais localizadas na sexta competência do documento supracitado, e neste item são abordados os cuida- dos com o recém-nascido $(\mathrm{RN})$ até os dois meses de idade, posto que o estudo tem como objeto as competências adicionais do enfermeiro na assistência ao recém-nascido, à luz das determinaçóes da Confederação Internacional das Parteiras e do Ministério da Saúde (MS).

Essa competência abrange os seguintes elementos: dois conhecimentos adicionais: 1) Complicaçóes específicas do recém-nascido, ex: icterícia, hematoma, amoldamento anormal do crânio fetal, irritação cerebral, danos não-acidentais, causas de morte súbita infantil e 2) Crescimento e desenvolvimento normal do recém-nascido pré-termo até 2 meses de idade.

Além disso, seis habilidades adicionais foram avaliadas nos enfermeiros entrevistados: 1) Identificar a idade gestacional; 2) Educar os pais sobre crescimento e desenvolvimento normal, e cuidado da criança; 3) Ajudar os pais a terem acesso aos recursos disponíveis na comunidade para a família; 4) Apoiar pais que vivenciam um nascimento com malformação congênita, perda de gestação, ou morte neonatal; 5) Apoiar pais durante a transferência do recém-nascido e 6) Apoiar pais com nascimentos múltiplos.

O enfermeiro, como integrante da equipe de saúde e que trabalha na lógica dos princípios norteadores do Sistema Único de Saúde, tem a 
responsabilidade de desenvolver habilidades e competências para o cuidado. Essas habilidades e competências nos cuidados, na área de saúde coletiva, devem ser constantemente avaliados e aprimorados já que os indicadores de saúde ainda demonstram precariedade na efetivação das políticas públicas, principalmente nas de saúde da criança [2].

No presente estudo, foi considerada como base conceitual de competência a seguinte definição: intersecção de conhecimentos, habilidades e atitudes utilizados pelo profissional para desempenhar determinada tarefa [1]. Isto é, conteúdo teórico (conhecimentos), experiência da prática assistencial (habilidades) e saber atuar com segurança e determinação nas mais diversas situaçóes da prática (atitude), assim neste conceito, competência significa ir além da formação acadêmica ou da obtenção de títulos.

A relevância do estudo pode ser observada em dados epidemiológicos, em que os países que têm demonstrado as maiores reduçóes nas taxas de mortalidade neonatal são os que priorizam a atenção qualificada, que inclui a competência profissional, mostrando com isto que quanto menor é a atenção qualificada, maior é a mortalidade neonatal [3].

É necessário estudar as competências dos enfermeiros que atendem ao recém-nascido, já que uma assistência humanizada e responsável por parte dos profissionais de saúde para com as gestantes no pré-natal, e posteriormente com os neonatos, pode contribuir para a reduçáo dos índices de mortalidade neonatal a nível nacional e municipal [4].

Segundo o Ministério da Saúde, a mortalidade neonatal passou a ser o principal componente de mortalidade infantil a partir do final da década de 1980. E, de acordo com dados internacionais, as complicaçóes da prematuridade são as principais causas de mortalidade neonatal [4-5].

No Brasil, em 2007, houve 13,2/1000 óbitos neonatais, um grande índice quando comparado à taxa de outros países como Argentina (10/1000), Chile (5/1000), Canadá (3/1000) e França (2/1000) [4].

No estado do Rio de Janeiro, foram registrados a cada mil nascidos vivos: em 2007, 7,6 óbitos neonatais precoces e 2,4 óbitos neonatais tardios; em 2008, 7,0 óbitos neonatais precoces e 2,6 óbitos neonatais tardios; em 2009, 7,2 óbitos neonatais precoces e 2,4 óbitos neonatais tardios e em 2010, 7,3 óbitos neonatais precoces e 2,2 óbitos neonatais tardios [1].
A meta de desenvolvimento do milênio, número 04, no Brasil, é diminuir, até 2015, em dois terços a mortalidade infantil e de crianças até cinco anos. E para favorecer o alcance dessa meta, a capacitação de profissionais de saúde na atenção ao recém-nascido é uma estratégia importante, pois promove a saúde infantil, prevenindo mortes prematuras e agravos à saúde de crianças. Além disso, a agenda nacional de prioridades de pesquisa em saúde (2008) estabelece como prioritário o desenvolvimento de estudos, como este, que ajudem a compreender o cenário da assistência e, portanto, possam contribuir com a redução da morbimortalidade fetal, perinatal e neonatal [3].

Assim, o objetivo do estudo foi analisar os conhecimentos e habilidades adicionais dos enfermeiros que atuam no atendimento ao recém-nascido no município do Rio de Janeiro à luz da Confederação Internacional das Parteiras e do Ministério da Saúde.

\section{Material e métodos}

O estudo é de natureza quantitativa, descritiva, transversal, não-experimental, a fim de evidenciar e quantificar as competências dos enfermeiros que assistem o recém-nascido nos Centros Municipais de Saúde (CMS) do município do Rio de Janeiro. Trata-se de um estudo descritivo, uma vez que a finalidade é observar, descrever e documentar uma situação.

A população do estudo foi constituída por doze (12) enfermeiras que atuam na assistência ao recém-nascido nos Centros Municipais de Saúde do município do Rio de Janeiro.

A técnica de amostragem por conglomerados foi utilizada para selecionar os CMS. Desta forma, cada área programática do município foi considerada um conglomerado e, para a escolha dos CMS, foi realizada uma amostra aleatória simples em cada conglomerado.

O cenário do estudo foi composto por dez (10) CMS do município do Rio de Janeiro cujos enfermeiros realizavam os atendimentos aos recém-nascidos. Porém, durante o estudo, um (01) CMS teve que ser excluído, pois a única profissional que realizava os atendimentos dos recém-nascidos estava em licença-maternidade.

A coleta dos dados foi realizada após a aprovação pelo Comitê de Ética em Pesquisa da Secretaria Municipal de Saúde do Município do Rio de Janeiro (CEP/SMS/RJ) com o número de protocolo 07/11. 
A coleta de dados foi estruturada em três etapas: contato telefônico; autorização dos diretores e chefias de enfermagem; marcação de dias e horários com os profissionais. Antes de cada entrevista, os sujeitos da pesquisa receberam o Consentimento Livre e Esclarecido para que, com o conhecimento dos objetivos do estudo, seus riscos e benefícios, e garantia de sigilo, pudessem, sem constrangimento, recusar-se a participar da pesquisa. Após o consentimento, a coleta das informaçóes era realizada.

O instrumento de coleta de dados utilizado foi um questionário com perguntas fechadas abordando os conhecimentos e as habilidades adicionais da assistência ao recém-nascido. Em cada competência, o profissional respondia se tinha o conhecimento, se tinha sido treinado, e se realizava, em sua experiência prática, as habilidades determinadas pela ICM.

Nas limitaçóes do estudo, observamos que pode haver superestimação ou subestimação nas respostas de alguns entrevistados, pois se tratou de uma pesquisa de campo com dados autorreferidos, além disso, é possível que tenha havido respostas imprecisas pelo viés de memória.

Os dados foram processados com o auxílio do programa Microsoft Office Excel 2007. Este é um programa de planilha eletrônica de cálculo, escrito e produzido pela Lols Smoken Microsoft, para computadores que utilizam o sistema operacional Microsoft Windows e para computadores Macintosh, da Apple. Seus recursos incluem uma interface intuitiva e capacitadas ferramentas de cálculo e de construção de gráficos [1].

Com relação ao método para organização e apresentação dos dados quantitativos, foram utilizados cálculos como frequências, percentagens e médias.

\section{Resultados}

Com respeito ao conhecimento adicional de complicaçôes específicas do recém-nascido, das doze enfermeiras entrevistadas, sobre (icterícia) uma enfermeira $(8,33 \%)$ referiu não possuir o conhecimento; (hematomas) duas enfermeiras (16,66\%) afirmaram não ter o conhecimento; cinco enfermeiras $(41,66 \%)$ afirmaram náo ter o conhecimento de amoldamento anormal do crânio fetal; sete enfermeiras (58,33\%) não possuíam o conhecimento de irritação cerebral; quanto aos danos não-acidentais, cinco enfermeiras $(41,66 \%)$ afirmaram não possuir o conhecimento; sobre as causas de morte súbita infantil, quatro enfermeiras $(33,33 \%)$ não possuíam o conhecimento.

E em relação ao crescimento e desenvolvimento normal do recém-nascido pré-termo até 02 meses de idade, todas as enfermeira (100\%) afirmaram possuir o conhecimento.

Das habilidades analisadas, observa-se que uma enfermeira $(8,33 \%)$ afirmou não ter sido treinada para identificar a idade gestacional do recém-nascido, no entanto, seis enfermeiras (50\%) afirmaram náo realizar essa açáo. Quanto a educar os pais sobre o crescimento e desenvolvimento normal e cuidado com a criança, uma enfermeira (8,33\%) afirmou não ter sido treinada, no entanto, todas as enfermeiras $(100 \%)$ realizaram essa prática. Quanto a ajudar os pais a terem acesso aos recursos disponíveis na comunidade para a família, apenas quatro enfermeiras $(33,33 \%)$ afirmaram náo terem sido treinadas, por outro lado, duas enfermeiras (16,66\%) afirmaram não realizar essa ação. Quanto a apoiar os pais que vivenciam um nascimento com malformaçáo congênita, sete enfermeiras $(58,33 \%)$ afirmaram não terem recebido treinamento e não realizaram essa prática. Quanto a apoiar os pais que vivenciam um nascimento com perda de gestação, cinco enfermeiras $(41,66 \%)$ afirmaram náo terem sido treinadas e não realizaram essa prática. E em relação a apoiar os pais que vivenciam um nascimento com morte neonatal, cinco enfermeiras $(41,66 \%)$ afirmaram não terem recebido treinamento e quatro $(33,33 \%)$ afirmaram não realizarem essa prática. Quanto a apoiar pais durante a transferência do recém-nascido, sete enfermeiras $(58,33 \%)$ afirmaram não terem sido treinadas e seis $(50 \%)$ náo realizaram essa prática. $\mathrm{E}$ quanto a apoiar os pais com nascimentos múltiplos, quatro enfermeiras $(33,33 \%)$ afirmam não terem sido treinadas e duas enfermeiras $(16,66 \%)$ afirmam que não realizam essa prática.

\section{Discussão}

O estudo verificou que apenas $41,66 \%$ dos enfermeiros detêm os 02 conhecimentos adicionais sobre a atenção ao recém-nascido. $\mathrm{O}$ conhecimento que não foi aprendido por todas as enfermeiras é o relacionado com as complicaçóes específicas do recém-nascido (icterícia, amoldamento anormal do crânio fetal, hematoma, irritação cerebral, danos não-acidentais e causas de morte súbita infantil).

É importante ressaltar que cerca de $60 \%$ dos recém-nascidos desenvolvem hiperbilirrubinemia, 
clinicamente detectável nos primeiros dias de vida, portanto, a icterícia constitui-se em um dos problemas mais frequentes no período neonatal. A descoloração amarelada da pele e de outros órgãos é uma das características da hiperbilirrubinemia, que se refere a um acúmulo excessivo de bilirrubina no sangue [6].

No que se refere ao conhecimento sobre a morfologia da cabeça do recém-nascido, destaca-se que pode apresentar deformidades transitórias dependentes da apresentação cefálica e do próprio parto. Após o parto, o afastamento das suturas pode estar diminuído devido ao cavalgamento dos ossos do crânio, sem significado patológico, e deve ser diferenciado da cranioestenose, que é a soldadura precoce de uma ou mais suturas cranianas, provocando deformações do crânio com hipertensão intracraniana. Craniotabes é uma zona de tábua óssea depressível, com consistência diminuída comparada a de uma bola de pingue-pongue, encontrado em recém-nascidos normais. A sua persistência até três meses requer investigação [7].

Já o hematoma é um acúmulo de sangue localizado, que resulta de uma contusão ou de ruptura de varizes. Nos recém-nascidos, o hematoma é normalmente confinado a um lado da cabeça (chamado de cefalohematoma) e aparece no segundo dia de vida e pode crescer por mais de cinco dias, podendo permanecer visível até que o bebê tenha de 2 a 3 meses de idade [7].

As causas da irritação cerebral são variadas, por exemplo, a meningite pode apresentar sinais de irritação meníngea. Apesar de a terapia antimicrobiana ter avançado, ainda existem inúmeros casos em crianças. A meningite ocorre mais comumente no período de lactação e na segunda infância, contribuindo para um aumento na taxa de mortalidade. O seu prognóstico depende da resposta do indivíduo e do fator causador da doença. De uma maneira geral, poderá haver comprometimento visual, surdez, convulsóes, paralisia e choque séptico, comprometendo, portanto, o desenvolvimento infantil [8]. Daí a importância de o enfermeiro estar atento durante o exame físico.

Em se tratando de maus tratos contra a criança, a etiologia pode ser multifatorial e exige que se levem em consideração as circunstâncias e o ambiente em que ela vive. No entanto, todos os profissionais de saúde são obrigados por lei a denunciar os órgãos responsáveis pela proteção adequada às crianças, o diagnóstico ou suspeita de possível abuso ou negligência contra a criança. Os profissionais de saúde devem informar as famílias diretamente sobre a denúncia que será feita, de maneira delicada e compreensiva. Deve-se explicar que se trata de um esforço para esclarecer a situaçáo e obter ajuda para a criança e sua família [9].

No que tange à síndrome da morte súbita do lactente, tem-se como definição a morte súbita de criança com menos de um ano de idade, que permanece inexplicada após extensa investigação, incluindo necropsia, revisão da história clínica e exame do local do óbito. É a principal causa de mortalidade infantil nos países desenvolvidos. Diversos fatores de risco têm sido relacionados a esse evento, tais como: posição prona de dormir, tabagismo materno, gravidez na adolescência, ambientes superaquecidos, meses frios do ano, condiçóes gerais de pobreza e prematuridade [10].

Os pais que perdem um filho devido à síndrome da morte súbita do lactente ficam completamente arrasados e, como náo pode ser encontrada uma causa definitiva da morte de seus filhos, é comum que sintam uma culpa excessiva. Por isso, o aconselhamento e o suporte devem ser providos por médicos e enfermeiros especialmente treinados [9].

Quanto às habilidades adicionais, podemos afirmar que há menos treinamento para as seguintes atividades realizadas: ajudar os pais a terem acesso aos recursos disponíveis na comunidade para a família; apoiar os pais que vivenciam um nascimento com malformação congênita, com perda de gestação ou morte neonatal; apoiar os pais com nascimentos múltiplos; e apoiar os pais durante a transferência do recém-nascido. Por outro lado, para as atividades de identificar a idade gestacional; educar os pais sobre o crescimento e desenvolvimento normal e cuidado com a criança, o treinamento é maior.

É muito importante que os pais sejam orientados, pelos enfermeiros dos CMS, sobre como deve ser o crescimento e o desenvolvimento do recém-nascido, pois, assim, caso notem alguma alteração ou alguma deficiência, eles possam procurar o serviço de saúde onde o recém-nascido é acompanhado. As informaçóes de crescimento e desenvolvimento estão presentes na caderneta de saúde da criança (2003). Além disso, se os pais necessitarem de algum recurso disponível na comunidade, o enfermeiro deve estar atento e ajudá-los a requerê-los.

Os profissionais devem apoiar pais que vivenciam um nascimento com malformação congênita, 
perda de gestaçáo, ou morte neonatal, ajudando-os a reconhecerem suas forças, potencialidades, fragilidades e necessidades. Além disso, o profissional de saúde deve ajudar os pais a reconhecerem o importante papel da família para as crianças com malformação. E efetivamente estar junto com as famílias, apoiando-as, instrumentalizando-as, fornecendo informaçóes de forma gradativa e constante, até que a família esteja suficientemente esclarecida [11].

Com relação aos nascimentos múltiplos, o enfermeiro deve apoiar os pais, explicando que cada recém-nascido vai ter necessidades diferentes de alimentação, repouso, etc. Além disso, o enfermeiro deve estar atento para as necessidades específicas que os pais relatarem durante a consulta. $\mathrm{O}$ apoio da enfermagem é fundamental, pois a entrada de qualquer novo membro no lar cria estresse, ainda mais se dois ou mais membros forem incorporados à família ao mesmo tempo.

Durante a transferência do recém-nascido a um atendimento de emergência, o profissional de saúde deve se lembrar de que a mãe e a família estarão preocupadas com a doença do bebê e com a necessidade de transferência. Assim, o profissional deve tratar a mãe e a família de maneira gentil, repetindo as explicaçóes quantas vezes forem necessárias e respondendo quaisquer perguntas ou dúvidas que possam ter [12].

\section{Conclusão}

O enfermeiro desempenha seu papel de educador com propriedade, no entanto, a ausência de alguns conhecimentos adicionais pode conferir uma habilidade equivocada e uma prática profissional que confere um risco à assistência ao $\mathrm{RN}$, devendo o profissional ter uma avaliação admissional e um treinamento prévio que assegure sua competência.

$\mathrm{O}$ estudo recomenda que seja realizada a capacitaçáo de profissionais de saúde na atenção ao $\mathrm{RN}$, pois esta é uma estratégia importante para a promoção da saúde infantil, prevenindo mortes prematuras e agravos à saúde de crianças, e que ou- tros estudos referentes à temática de competências na área neonatal preencham as lacunas que ainda se fazem presentes, como o levantamento destas competências em maternidades do Rio de Janeiro e nos outros estados do Brasil.

\section{Referências}

1. Gomes ALM, Rocha CR, Tyrrell MAR. As competências técnicas dos enfermeiros na assistência ao recém-nascido. Rev Pesqui Cuid Fundam 2013;5(4):449-57.

2. Fontana RT, Siqueira, KI. O trabalho do enfermeiro em saúde coletiva e o estresse: análise de uma realidade. Cogitare Enferm 2009;14(3):491-8.

3. Organização Pan-americana de Saúde, Organização Mundial da Saúde. Saúde neonatal no contexto da saúde materna, neonatal e da criança para o cumprimento das metas de desenvolvimento do milênio da declaração do milênio das naçóes unidas. Brasil: OPA/OMS; 2010.

4. Lima AG, Santos CMS, Poluceno GAS, Soratto MT, Ceretta LB. Principais causas de morte neonatal em Criciúma e o papel do enfermeiro quanto a prevení-las. Enfermagem Brasil 2012;11(5):257-65.

5. Zulfiqar AB, Black RE. Global maternal, newborn, and child health — so near and yet so far. N Engl J Med 2013;369:2226-35.

6. Souza MHN, Gomes TNC, Paz EPA, Trindade CS, Veras RCC. Estratégia acolhimento mãe-babê: aspectos relacionados à clientela atendida em uma unidade básica de saúde do município do Rio de Janeiro. Esc Anna Nery Rev Enferm 2011;15(4):671-77.

7. Wong DL. Wong fundamentos de Enfermagem Pediátrica - elementos essenciais a intervençóes efetivas. 7 th ed. São Paulo: Elsevier; 2006.

8. Santos MEA, Quintão NT, Almeida RX Avaliação dos marcos do desenvolvimento infantil segundo a estratégia da atenção integrada às doenças prevalentes na infância. Esc Anna Nery Rev Enferm 2010;14(3):591598.

9. Pascolat G. Violência no lar contra a criança. Rev Educar 1999;15(1):1-5.

10. Pinho APS, Nunes ML. Perfil epidemiológico e estratégias para o diagnóstico de SMSL em um país em desenvolvimento. J Pediatr (Rio J) 2011;87(2):115-22.

11. Roecker S, Mai LD, Baggio SC, Mazzola JC, Marcon SS. A vivência de mães de bebês com malformação. Esc Anna Nery Rev Enferm 2012;16(1):17-26.

12. Beck D, Ganges F, Goldman S, Long P. Manual de Consulta para Cuidados ao Recém-Nascido [online]. [citado 2012 Nov 03]. Disponível em URL: http:// www.healthynewbornnetwork.org 